\title{
Chemistry of 1,2-Anhydro Sugars
}

\author{
Gefei Li, Masato Noguchi, Kazunari Serizawa, and Shin-ichiro Shoda* \\ This paper is dedicated to the memory of my mentor, Professor Teruaki Mukaiyama, the originator \\ of the Lewis acid-catalyzed Aldol reaction.
}

\begin{abstract}
The 1,2-anhydro sugars are a class of valuable and versatile intermediates in carbohydrate chemistry. In the first part of this article, a review is given on preparation methods of 1,2-anhydro sugars that are suitably protected. Protected 1,2-anhydro sugars have been widely used as glycosyl donors for the synthesis of glycosyl compounds such as oligosaccharides and nucleosides. In the second part, a brief history and the chemistry of unprotected 1,2-anhydro sugars is described. In the past few years, our research group has developed protection-free methods for synthesis of glycosyl compounds through unprotected 1,2-anhydro sugars as reactive intermediates based on the concept of 'direct anomeric activation'. In this article, the one-step preparation of glycosyl compounds such as thioglycoside derivatives and glycosyl azides by using formamidinium-type dehydrating agents is presented. Furthermore, the initial results on the first detection of unprotected 1,2-anhydro sugar intermediates by NMR measurements are shown along with their full structure characterizations.
\end{abstract}

Keywords: 1,2-Anhydro sugar · 2-Chloro-1,3-dimethylimidazolinium chloride (DMC) · Direct anomeric activation - Glycosyl azide · Glycosyl Bunte-salt

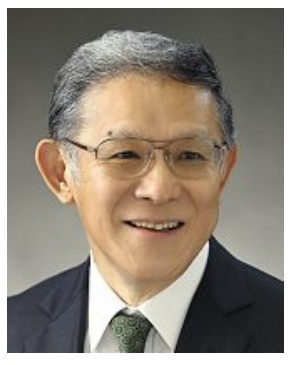

\section{Shin-ichiro Shoda}

was born in Tokyo, Japan in 1953. He received his $\mathrm{PhD}$ degree from the University of Tokyo in 1981 under the supervision of Prof. Teruaki Mukaiyama, where he developed the glycosyl fluoride method as a novel glycosylating technology. After spending three years working as an Assistant Professor at the University of Tokyo, he conducted his postdoctoral fellowship at ETH-Zurich (from 1984 till 1986) with Prof. Dieter Seebach. In 1986, he moved to Tohoku University and joined the lab of Prof. Shiro Kobayashi in the field of polymer synthesis, where he developed new chemo-enzymatic glycosylations by using glycosyl fluorides and sugar oxazolines as glycosyl donors. In 1999, he was promoted to Full Professor at Tohoku University (Functional Macromolecular Chemistry Laboratory). His research interests include development of novel glycosylations and precision synthesis of functional oligo- and polysaccharides. He has been a Member of the Research Center for Science System at the Japan Society for the Promotion of

\footnotetext{
${ }^{\star}$ Correspondence: Prof. S. Shoda Department of Biomolecular Engineering Graduate School of Engineering, Tohoku University Aoba, Sendai 980-8579, Japan

E-mail: shinshoda@tcn-catv.ne.jp
}

Science (from 2003 till 2005). He received the Award of the Chemical Society of Japan for Young Chemists (1986), Science and Technology Institute Award for New Invention (1993), the Cellulose Society of Japan Award (2002), and Synthetic Organic Chemistry Award, Japan (2013).

\section{Introduction}

The 1,2-anhydro sugars possess a skeleton where a six-membered pyranose ring and a three-membered oxirane ring are fused sharing the anomeric position and the $\mathrm{C} 2$ position. In the history of carbohydrate chemistry, 1,2-anhydro sugars have established their firm position as useful intermediates for the preparation of $O-, C$ and $N$-glycosyl compounds. The existence of a distorted linkage makes 1,2-anhydro sugars extremely reactive such that ringopening reactions takes place easily at the anomeric position by the attack of a nucleophile, generating a free $\mathrm{OH}$ at the $\mathrm{C} 2$ position. In 1922, Brigl reported the synthesis of 1,2-anhydro- $\alpha$-D-glucopyranose triacetate ${ }^{[1]}$ and since then, this compound has been commonly called 'Brigl's anhydride' among carbohydrate chemists. However, the application of 1,2-anhydro sugars did not gain much attention in early times, because the synthesis via the distorted cyclic structure presented several technical hurdles. It was not until the 1990s that the extensive use of protected 1,2-anhydro sugars emerged in the synthesis of complex oligosaccharides and $N$-glycosides. A large number of examples using protected 1,2-anhydro sugars as intermediates have been reported by Danishefsky's group ${ }^{[2]}$ based on the discovery of the easy access to protected 1,2-anhydro sugars by the epoxidation of the corresponding glycals (cyclic enol ethers of sugars having a double bond between the anomeric position and the $\mathrm{C} 2$ position) with a mild oxidizing agent, dimethyldioxirane (DMDO). In addition to the development of 1,2-anhydro pyranoses (six-membered ring), it has also been shown that protected 1,2-anhydro furanoses (five-membered ring) are promising glycosyl donors for the synthesis of nucleosides.

Another turning point of 1,2-anhydro sugar chemistry was the discovery of the direct conversion of unprotected sugars to 1,6-anhydro sugars in our research group by using a formamidinium-type dehydrating agent, 2-chloro-1,3-dimethylimidazolinium chloride (DMC), where unprotected 1,2-anhydro sugars were speculated as the key intermediates towards the intramolecular nucleophilic substitution of the hydroxy group from the 6 position. ${ }^{[3]}$ These findings triggered the protection-free synthesis of glycosyl compounds in aqueous media through unprotected 1,2-anhydro sugars as reactive intermediates. Furthermore, in 2017, we firstly detected the unprotected 1,2-anhydro species by low-temperature NMR spectroscopy that allowed optimization of protection-free synthesis of glycosyl compounds. ${ }^{[4]}$ In this review, an account is given of developments in 1,2-anhydro sugar chemistry, including synthetic methodologies of protected and unprotected 1,2-anhydro sugars, and their applications in the synthesis of oligosaccharides and glycoconjugates. 


\section{Synthesis of Protected 1,2-Anhydro Sugars}

Currently, two methods have been commonly employed for the synthesis of protected 1,2-anhydro sugars. In the first method, the intramolecular nucleophilic attack of an oxyanion to the anomeric carbon atom leads to the formation of a 1,2-anhydro skeleton. This strategy is straightforward where the logic of synthesis is the same as that of the synthesis of ethylene oxide (oxirane) through the intramolecular substitution of a carbon having a good leaving group by a hydroxyl group on the adjacent carbon atom. In the traditional method of Brigl, glucopyranose pentaacetate (1) is treated with phosphorus pentachloride in carbon tetrachloride to yield acetylated 2-O-trichloroacetyl-glucosyl chloride (2, Scheme 1).[1] Thereafter, the resulting chloride $\mathbf{2}$ is treated with ammonia to achieve the selective deprotection of the trichloroacetyl group, delivering glucosyl chloride $\mathbf{3}$ having a free hydroxy group on the $\mathrm{C} 2$ position. Finally, the intramolecular nucleophilic substitution of the $\beta$-isomer of $\mathbf{3}$, which is promoted by further reaction with ammonia, gives Brigl's anhydride 4.

By using a similar synthetic strategy using an intramolecular substitution, Sondheimer and co-workers synthesized 1,2-anhydro mannopyranose 5 through the ammonia-triggered reaction of 3,4,6-tri$O$-benzyl- $\alpha$-mannopyranosyl chloride (6, Scheme 2A). ${ }^{[5]}$ Potassium tert-butoxidemediated intramolecular ring closure of $\beta$-galactopyranosyl fluoride 7 also gave 1,2-anhydro galactose $\mathbf{8}$ quantitatively via the 2-OH intermediate (Scheme 2B). ${ }^{66]}$ The alternative pathway of intramolecular substitution consists of the introduction of a leaving group at $\mathrm{C} 2$ and the subsequent ring closure. Treatment of 1-hydroxy sugar 9 bearing a $p$-toluensulfonyl (Ts) group at $\mathrm{C} 2$ with potassium tert-butoxide gave the corresponding 1,2-anhydro sugar $\mathbf{1 0}$ in good yield (Scheme 2C). ${ }^{[7]}$

The second method for the synthesis of 1,2-anhydro sugar $\mathbf{1 1}$ is the direct epoxidation of the corresponding glycals $\mathbf{1 2}$ by using oxidants such as dimethyldioxirane (DMDO) (Scheme 3). Inspired by Murray's work, ${ }^{[8]}$ Danishefsky and co-workers developed a simple method for the preparation of 1,2-anhydro sugars via DMDO-mediated epoxidation of protected glycals. When 3,4,6-tri- $O$-benzyl glucal and 3,4,6-tri- $O$ - $t$-butyldimethylsilyl glucal were used as starting materials, the reactions proceeded stereoselectively, affording gluco-type epoxide derivatives. However, the use of 3,4,6-tri- $O$-acetyl glucal gave a mixture of gluco-type and manno-type products. ${ }^{[2]}$ The studies that followed focused on the alternatives to
DMDO, because the challenge was how to scale up the synthesis using limited amounts of DMDO. A one-step epoxidation of glycals using $m$-chloroperoxybenzoic acid (mCPBA) and potassium fluoride (KF) in anhydrous dichloromethane was demonstrated by Chiappe and co-workers. The epoxidation of 3,4,6-tri- $O$-acetyl glucal preferably gave gluco-type epoxide by using this synthetic method with low yield $(25 \%$, gluco:manno $=8: 2) .{ }^{[9]}$ Perfluoro-cis-2,3-dialkyloxaziridines were also reported as oxidant in the epoxidation of glycals, giving rise to a mixture of diastereomers. ${ }^{[10]}$ Moreover, treatment of 3,4,6-tri-O-benzyl-D-glucal with diaryl sulfoxide and triflic anhydride $\left(\mathrm{Tf}_{2} \mathrm{O}\right) \mathrm{se}$ lectively gives the corresponding 1,2-anhydro glucose derivative in the presence of triethylamine and methanol.[11] It should be noted that the epoxidation occurs only after the addition of nucleophile (i.e. $\mathrm{MeOH})$ to activate the glycal intermediate. Since dioxiranes can be generated in situ from the reaction of ketones and potassium peroxymonosulfate (oxone), Dondoni and co-workers demonstrated a multigram epoxidation of 3,4,6-tri- $O$-benzyl-D- glucal (99\% yield, gluco-type only), and 3,4,6-tri- $O$-benzyl D-galactal (99\% yield, galacto-type only) in a biphasic system. The DMDO-epoxidation of 3,4,6-tri- $O$ acetyl glucal selectively gave gluco-type epoxide by oxone with high yield in large scale $(87 \%$ yield, gluco:manno $=7: 1) \cdot{ }^{[12}$ In addition, reactions using a metalloporphyrin catalyst were investigated. Che and co-workers ${ }^{[13]}$ employed ruthenium or manganese porphyrin as a catalyst for the epoxidation of glycals, affording an anomeric mixture of the corresponding 1,2-anhydro sugars. The poly(ethylene glycol)-supported metalloporphyrin was also investigated by the same research group, achieving a 99\% conversion from $O$-acetyl-D-glucal with high diastereoselectivity ( gluco:manno $=9: 1) .{ }^{[14]}$

\section{Protected 1,2-Anhydro Sugars as Glycosyl Donors}

\subsection{O-Glycosylation}

In the 1950s, Lemieux and Huber used Brigl's anhydride 4 for the first chemical synthesis of sucrose $\mathbf{1 3}$ (Scheme 4) and
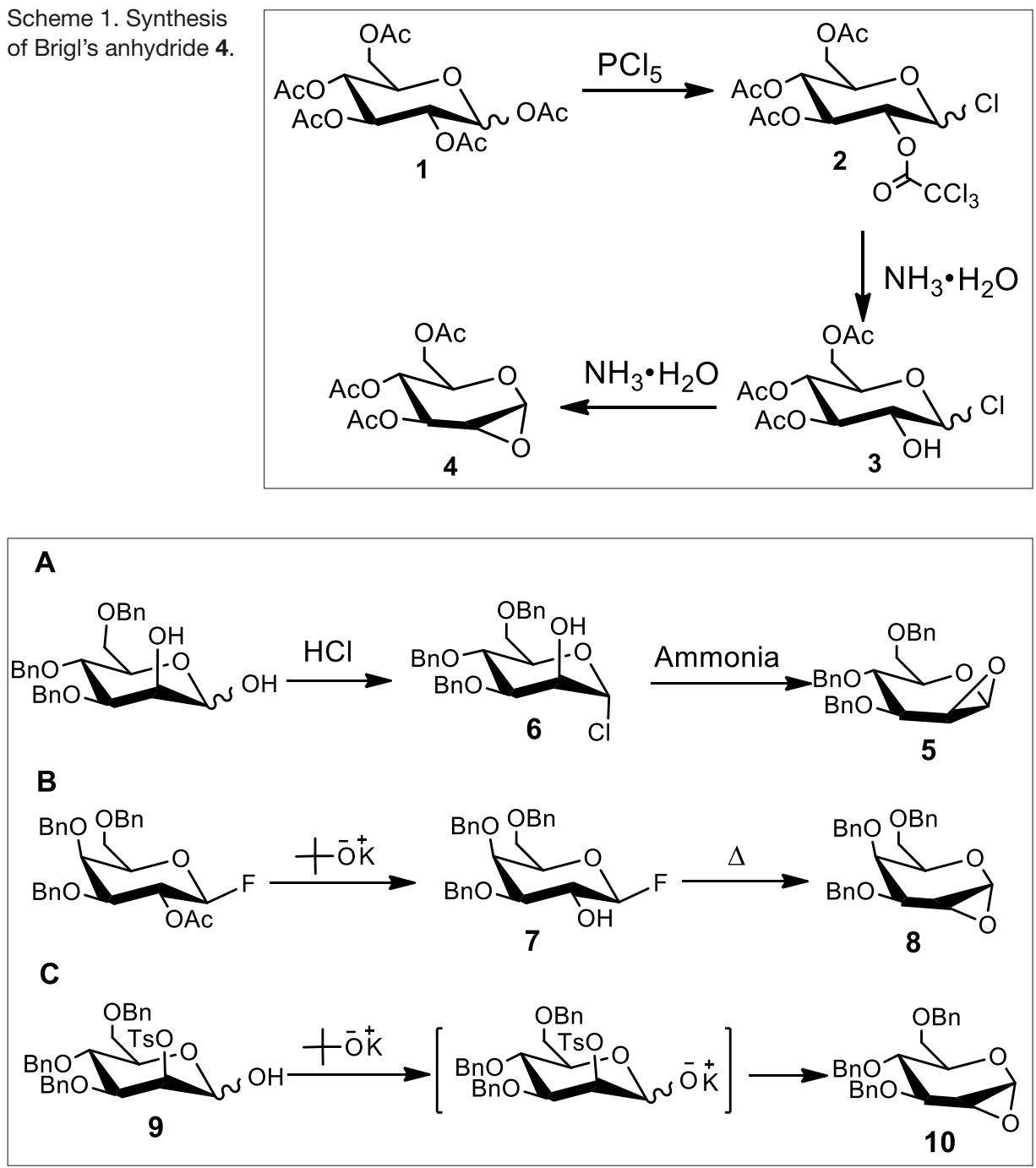

Scheme 2. Synthesis of protected 1,2-anhydro sugars via intramolecular nucleophilic substitution. 


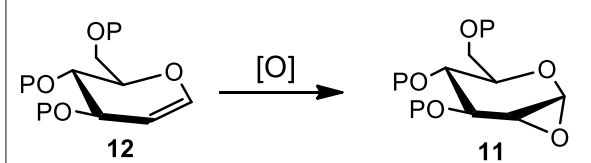

$P: A c, B n, M e, T E S .$.

[O]: DMDO, mCPBA/KF, $\mathrm{Ar}_{2} \mathrm{SO} / \mathrm{Tf}_{2} \mathrm{O} / \mathrm{Et}_{3} \mathrm{~N} / \mathrm{MeOH}$, Ruthenium or manganese Porphyrin

Scheme 3. Synthesis of protected 1,2-anhydro sugars via oxidation of glycals.

maltose. ${ }^{[15]}$ However, the mechanism for the $\alpha$-selective glycosidic bond formation was not clear. In the absence of acceptors, treatment of Brigl's anhydride with hot alkali led to the formation of levoglucosan (1,6-anhydro glucose). ${ }^{[16]}$ These reports clearly revealed that 1,2-anhydro sugars are useful intermediates for $O$-glycosylation.

From the late 80 s to 90 s, Danishefsky and co-workers reported several works with regard to the $O$-glycosylation using 1,2-anhydro sugars including solid-phase oligosaccharide synthesis. ${ }^{[2,17]}$ In contrast to Lemieux's results, the $\beta$-linked oligosaccharides $\mathbf{1 4}$ were stereoselectively prepared from per- $O$-benzylated 1,2-anhydro sugars (10 and $\mathbf{1 5}$ ) in the presence of multi-equivalents of zinc chloride (Scheme 5).
Based on Danishefsky's strategy, Kong and co-workers prepared glycopeptides via stereoselective $O$-glycosylation of L-serine. ${ }^{[18]}$ Some bioactive oligosaccharides and glycoconjugates such as tetrameric arabinogalactans ${ }^{[19]}$ were also successfully synthesized by the Lewis acid-assisted one monosaccharide-elongation process using 1,2-anhydro sugars as glycosyl donors. In addition, the important substrates for glycosyl transferases, UDP-sugars, have been prepared by the direct coupling of 1,2-anhydro sugars with uridine diphosphate. ${ }^{[20]}$

Advanced activation strategies have been developed for the stereocontrolled $O$-glycosylation using protected 1,2-anhydro sugars as glycosyl donors. In principle, opening of an oxirane ring is promoted by the action of an acid catalyst. However, side reactions such as hydrolysis as well as low stereoselectivity are the concerns of using strong acids. In using 1,2-anhydro sugars as a glycosyl donor, $\mathrm{Yu}$ and co-workers disclosed that an improved yield could be obtained with $\mathrm{Ph}_{3}$ PAuOTf catalyst instead of anhydrous zinc chloride. [21] Boronic esters $\mathbf{1 6}$ have been proposed for the stereoselective glycosylation based on an intramolecular aglycon delivery strategy (Scheme 6). Takahashi and Toshima and co-workers $^{[22]}$ used a glycosyl-acceptor-derived

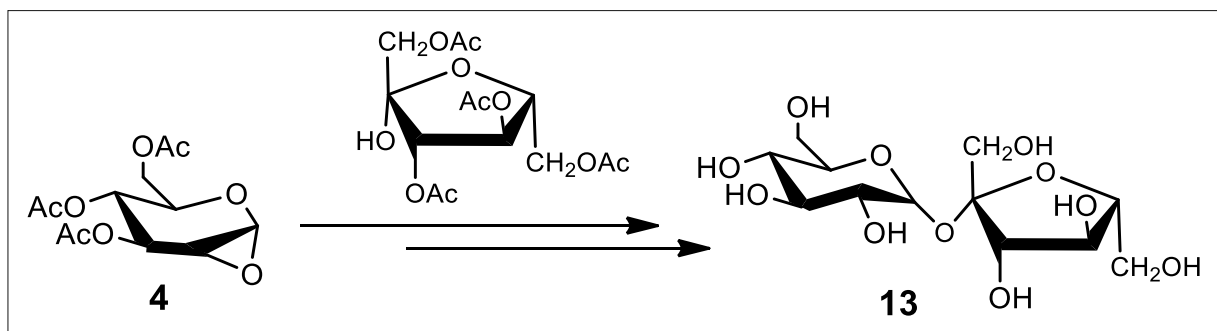

Scheme 4. First chemical synthesis of sucrose using Brigl's anhydride.

Scheme 5. Synthesis of $\beta$-linked oligosaccharides using per-Obenzolyated 1,2-anhydro sugars.

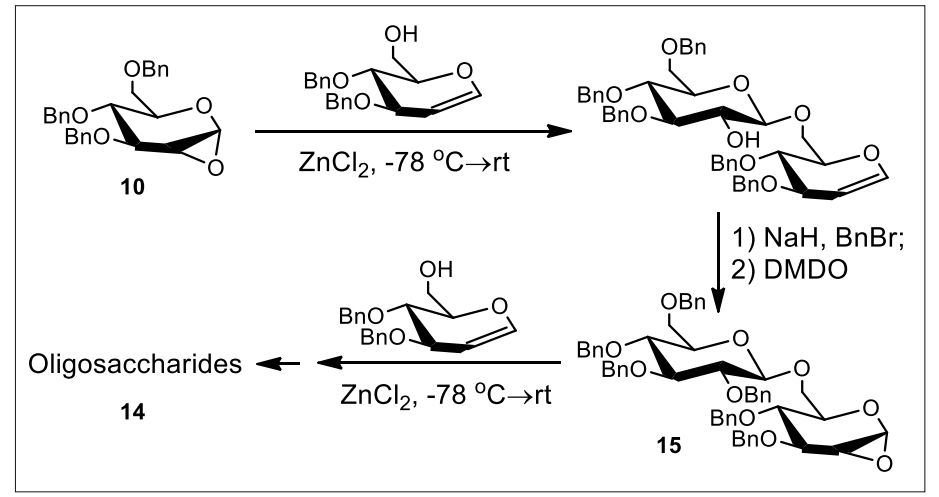

Scheme 6. An $\alpha$-selective glycosylation using intramolecular aglycon delivery strategy.

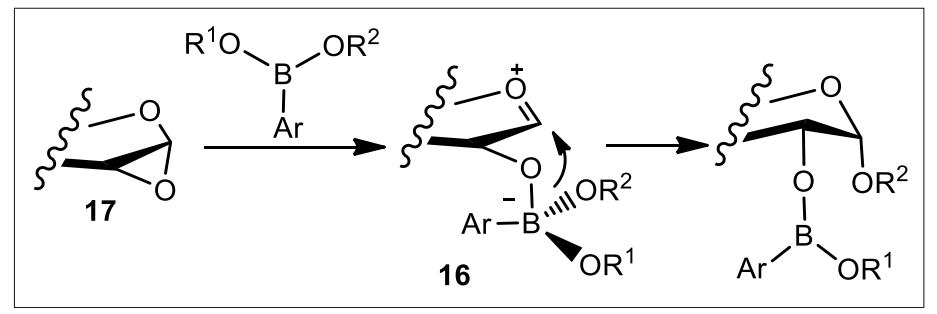

boronic ester to activate protected 1,2-anhydro glycosyl donors 17, affording the corresponding 1,2-cis-glycosides in high yields under mild reaction conditions. Aryl boronic acids were also reported as an aryl source to produce phenolic glycosides via the aromatic $O$-glycosylation of 1,2-anhydro sugars in the presence of a base. ${ }^{[23]}$ Furthermore, tunable stereoselectivity was found in the glycosylation of 1,2-anhydro sugars with phenol derivatives by varying the metal ion of the base. ${ }^{[24]}$

\subsection{C-Glycosylation}

Another important application of 1,2-anhydro sugars is the preparation of $C$-glycosides. In 2017, Yu and co-workers reviewed $C$-glycoside syntheses with 1,2-anhydro sugars as glycosyl donors. [25] Ring opening of 1,2-anhydro sugars for $C$-glycosylation often relies on the participation of an organometallic $C$-nucleophilic acceptor under electrophilic/cationic or radical pathways. Reactions of 1,2-anhydro sugars with organolithium ${ }^{[26]}$ and Grignard reagents ${ }^{[27]}$ usually lead to varied stereoselectivity depending on the substrates and reaction conditions (Scheme 7A). Organoaluminum, -zinc, -boron and -zirconium prefer to give 1,2-cis $C$-glycosides $\mathbf{1 8}^{[28]}$ (Scheme 7B), whereas reactions of organocuprates (Gilman reagent) ${ }^{[29]}$ favor 1,2-trans $C$-glycosylation (Scheme 7C). Treatment of 1,2-anhydro sugars with metal reagents such as $\mathrm{SmI}_{2}^{[30]}$ and $\mathrm{CP}_{2} \mathrm{TiCl}_{2}{ }^{[31]}$ gives anomeric radicals for $\alpha$-selective $C$-glycosylation (Scheme 7D).

Walczak and co-workers ${ }^{[32]}$ have described a novel approach for the stereoselective synthesis of $C$-aryl glycosides $\mathbf{1 9}$. In their method, the $O$-benzyl 1,2-anhydro sugar 14 is activated with $\mathrm{Bu}_{3} \mathrm{SnMgMe}$, giving rise to a glycosyl stannane species 20 which couples with aryl halides through a Pd-catalyzed highly stereoretentive cross-coupling reaction (Scheme 8).

\subsection{N- and S-Glycosylation}

Not limited to oligosaccharide syntheses, Danishefsky and co-workers used protected 1,2-anhydro sugars as glycosyl donors for the synthesis of nucleosides $\mathbf{2 1}$ via the $\mathrm{ZnCl}_{2}$ mediated $\mathrm{N}$-glycosylation (Scheme 9).[33] Thereafter, Kong and co-workers revealed that the reaction of 1,2-anhydro-5- $O$-acetyl-3- $O$-benzyl- $\alpha$-Dxylofuranoside with a trimethylsilylated thymine derivative in the absence of Lewis acid afforded $\mathrm{N}$-xylofuranosyl thymidine in good yields. ${ }^{[34]}$ Recently, the azidation of $O$-benzyl 1,2-anhydro sugars with sodium azide in acetonitrile by using a catalytic amount of ceric ammonium nitrate has been demonstrated, giving rise to $\beta$-glycosyl azides in good yields. ${ }^{[35]}$ 


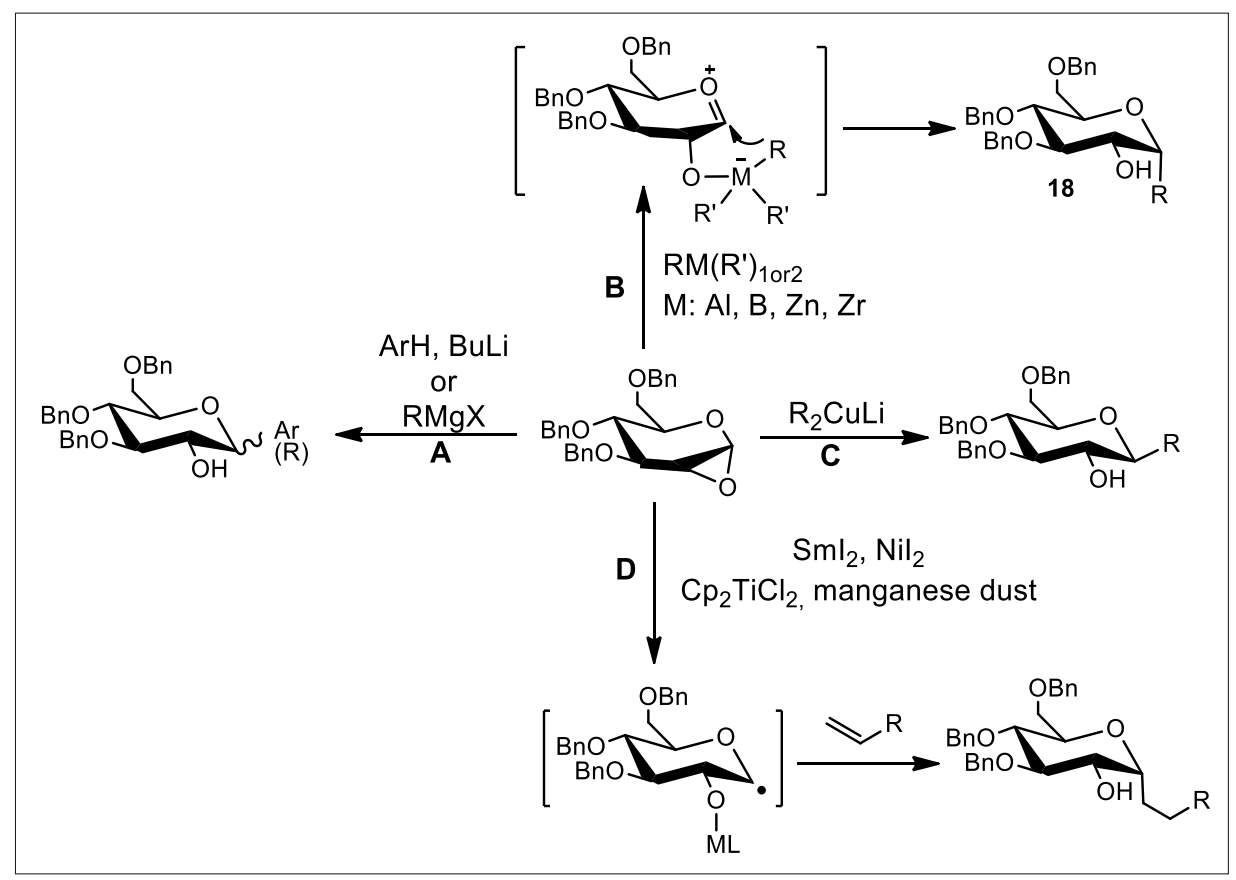

Scheme 7. C-Glycosylation using protected 1,2-anhydro sugars as glycosyl donors.

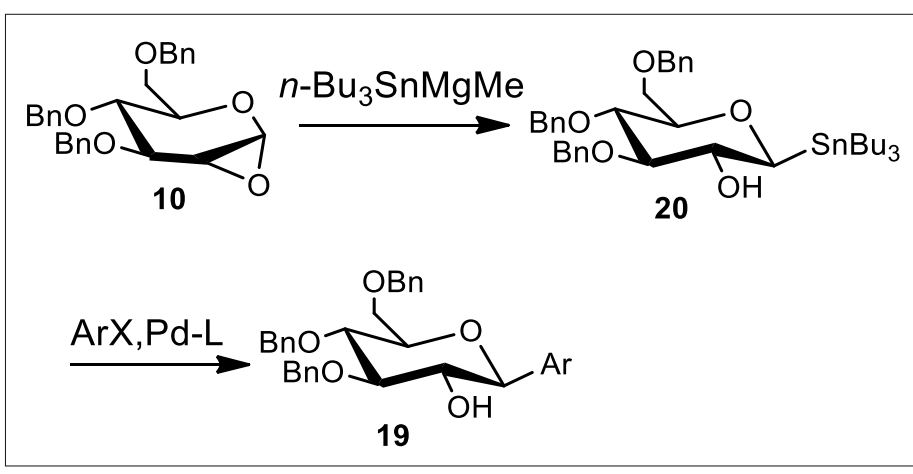

Scheme 8. Synthesis of glycosyl stannanes from 1,2-anhydrosugars toward $\mathrm{C}-\mathrm{C}$ coupling.

The 1,2-anhydro sugars have also been used as efficient glycosyl donors for $S$-glycosylation. The TMSOTf-catalyzed ring opening of a protected 1,2-anhydro sugar 10 with bis(trimethylsilyl) sulfide led to the formation of $\alpha$-glucosyl thiol 22 as the major product (Scheme 10A). ${ }^{[36]}$ The oxirane ring-opening glycosylation reaction between $\mathbf{1 0}$ and $N$-acetyl-L-cysteine methyl ester produced the cysteine thioglycoside $\mathbf{2 3}$ in the presence of Lewis acid (Scheme 10B). [37] Recently, treatment of 10 with $\mathrm{Et}_{2} \mathrm{NH}$ and $\mathrm{CS}_{2}$ enabled the in situ generation of glycosyl dithiocarbamates 24 (Scheme 10C), which could be acti- vated by copper triflate for the $\beta$-selective $O$-glycosylation. ${ }^{[38]}$

\subsection{Other Reactions Using Protected 1,2-Anhydro Sugars as Glycosyl Donors}

Protected 1,2-anhydro sugars have also been documented in the synthesis of some special carbohydrate derivatives. Glycosyl fluorides are obtained by the treatment of protected 1,2-anhydro sugars with excess amounts of tetrabutylammonium fluoride. ${ }^{[39]}$ Hydride addition to endocyclic 1,2-anhydro sugars offered an efficient route to differentially protected

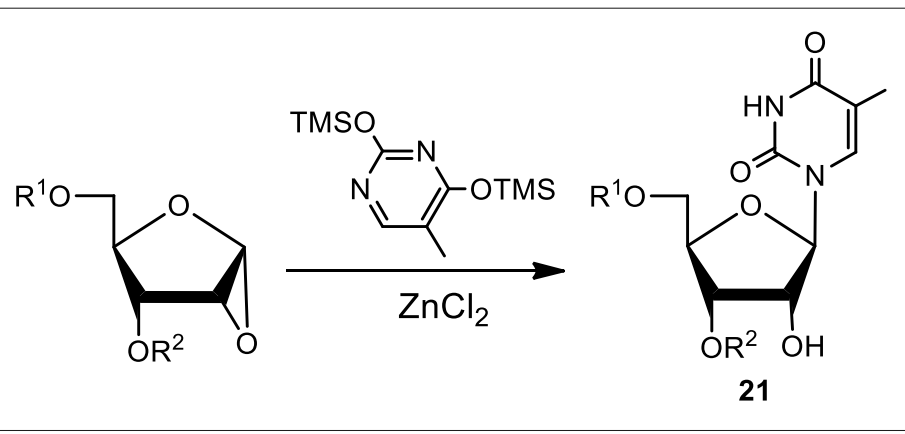

Scheme 9. Synthesis of nucleosides using 1,2-anhydro-sugar donors. 1-deoxy sugars. ${ }^{[40]}$ Reactions with carbon nucleophiles such as Grignard reagents with inversion of configuration produces L-sugars in a pyranosidic form. [41] Some carbohydrate-fused heterocycles $\mathbf{2 5}$ such as cis-1,2-fused 1,3-oxathiolan-, 1,3-oxaselenolan-, and 1,3-oxazolidin-2-imine carbohydrate derivatives have been prepared by treatment of 1,2-anhydro sugars 26 with potassium thiocyanate, potassium selenocyanate, and sodium cyanamide, respectively (Scheme 11). ${ }^{[42]}$

\section{History of Unprotected 1,2-Anhydro Sugars}

In contrast to reports on protected sugar derivatives, papers on unprotected 1,2-anhydro sugars are few and even the existence of these compounds has been wrapped in mystery for nearly a hundred years. In 1920, Pictet and Castan claimed the synthesis of an unprotected 1,2-anhydro sugar. ${ }^{[43]}$ However, Brigl disputed their results in 1929. [44] Since then, unprotected 1,2-anhydro sugars have only been recognized as undetectable intermediates in the alkaline hydrolysis of phenyl $\beta$-glucoside ${ }^{[45]}$ or alcoholysis of $\beta$-glucosyl fluoride in the presence of a strong base, sodium methoxide. ${ }^{[46]}$ Thus, the use of unprotected 1,2-anhydro sugars as intermediates for protection-free glycosylation has been greatly overlooked.

Our investigation of unprotected 1,2-anhydro sugars originates from the direct synthesis of sugar oxazolines, bicyclic sugar derivatives having a fused structure of a six-membered pyranose ring and a fivemembered oxazoline ring, in aqueous media. In traditional carbohydrate chemistry, the synthesis of sugar oxazoline derivatives necessitates a tedious process involving carefully designed protection/deprotection approaches. To develop an environmentally benign process, we tried to find a new method for the preparation of sugar oxazolines without using protecting groups, and finally discovered a one-step protocol by using a formamidinium-type dehydrating agent in aqueous media. ${ }^{[47]}$ Thereafter, this dehydrating agent was subjected to reaction with glucose, leading to the first one-step synthesis of 1,6-anhydro sugars 27 from unprotected sugars (Scheme 12). ${ }^{[3]}$ Interestingly, the reaction with 2-deoxy sugars did not proceed, strongly suggesting that the 1,2-anhydro sugar 29 is the key intermediate for the intramolecular substitution of the hydroxy group from the 6 position. Since 2009, the unprotected 1,2-anhydro sugars generated by the direct treatment of native sugars with 2-chloro-1,3-dimethylimidazolinium chloride (DMC) have become widely used among many carbohydrate chemists in the synthesis of glycosyl compounds such as 
Scheme 10. Ring opening of 1,2-anhydro sugars leading to S-glycosides.

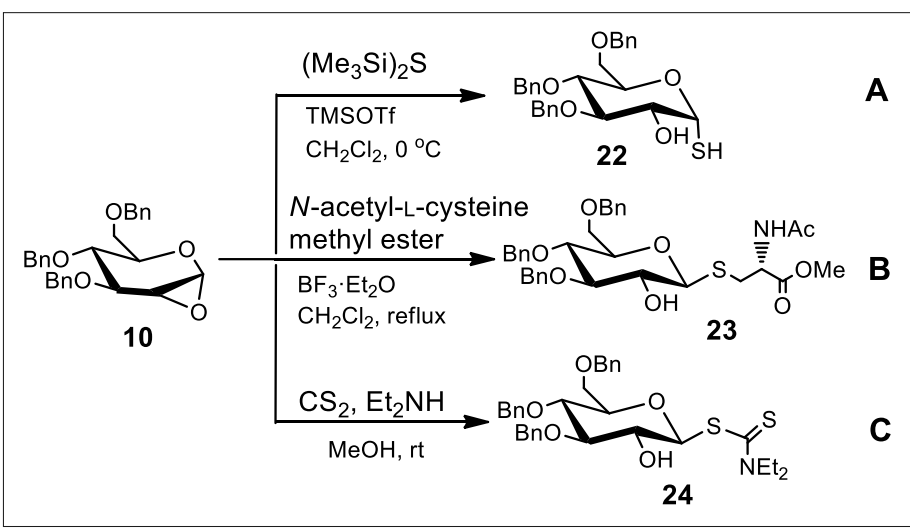

Scheme 11. Reaction of 1,2-anhydroglucopyranose with KXCN under the catalysis of Lewis acid.

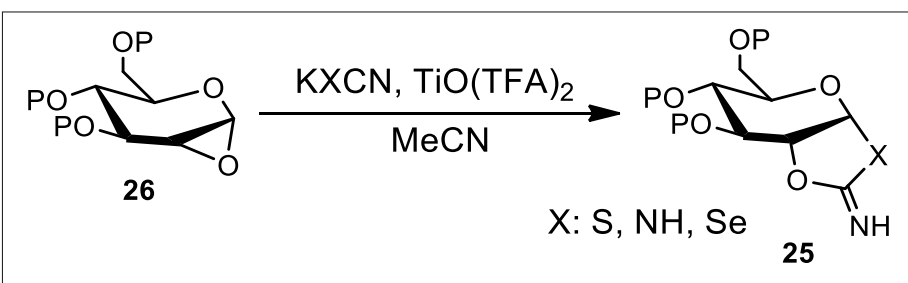

glycosyl azides and thioglycosides (direct anomeric activation).

\subsection{Unprotected 1,2-Anhydro Sugars as Reactive Intermediates}

With the direct anomeric activation technique using DMC, subsequent research focused on the selection of appropriate nucleophiles for glycosylation in aqueous media through unprotected 1,2-anhydro sugar intermediates (Scheme 13). In principle, glycosidic compounds can be regarded as dehydrative condensation products between hemiacetals and aglycons. An intermolecular dehydrative condensation is, however, extremely disadvantageous in water due to the large negative values of standard Gibbs energy of formation of glycosidic bonds. Our strategy for dehydrative condensation in water can be achieved via two elemental reactions: an intramolecular dehydrative condensation (Step 1) and the addition of a nucleophile to the anomeric center (Step 2). ${ }^{[48]}$ The first step has an entropical favorability over intermolecular reactions, and the second step does not require any dehydrative property because it has already been achieved in the first step. Hence, the selection of an appropriate nucleophile displays a key factor for the second intermolecular addition which would be affected by the competitive reactions from intramolecular 1,6-cyclization and hydrolysis (Scheme 13).

In the following years, a series of useful nucleophiles were discovered in our group for the aqueous glycosylation re-
Scheme 12. Direct synthesis of 1,6-anhydro sugars from unprotected glycopyranoses by using 2-chloro-1,3-dimethyl-imidazolinium chloride (DMC).

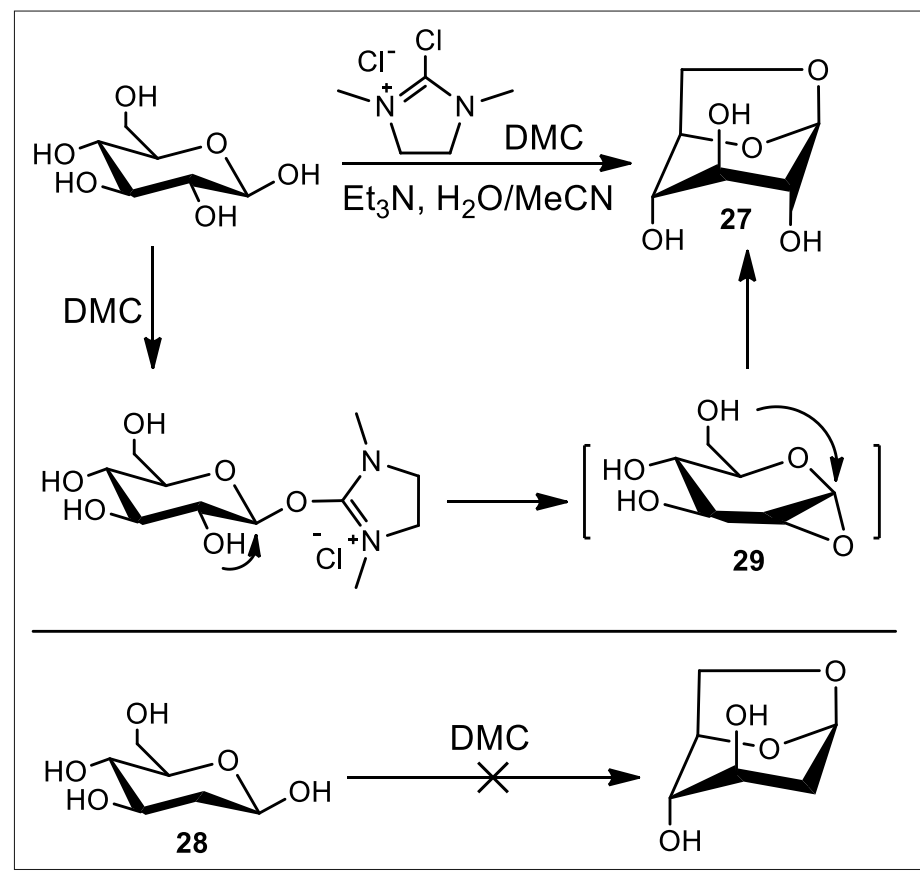

actions via the unprotected 1,2-anhydro sugar intermediates in the presence of DMC. In addition, a newly designed dehydrative condensing agent, 2-chloro-1,3-dimethyl-1 $H$-benzimidazol-3-ium chloride (CDMBI) was employed to overcome some disadvantages derived from hygroscopic DMC. ${ }^{[49]}$ In all cases, the nucleophilic opening of the oxirane ring occurs via anomeric attack to give the $\beta$-glycosides in a stereoselective manner. We began the study with the addition of aryl thiols ${ }^{[50]}$ to the reaction mixture of sugars and DMC for the synthesis of a range of $S$-aryl glycosides 30 (Scheme 14A). Thereafter, we continued this work and proposed a mechanism for the synthesis of various 2-pyridyl 1-thioglycosides ${ }^{[51]}$ and direct introduction of a detachable fluorescent tag (4-methyl-7-thioumbelliferyl group $)^{[52]}$ to the anomeric center of unprotected sugars. It should be highlighted that, according to the present method, not only unprotected monosaccharides but also unprotected oligosaccharides can be converted to the corresponding $S$-glycosyl derivatives, which would greatly expand the utility of aryl 1-thioglycosides in carbohydrate chemistry. This protocol was adopted by the groups of Winssinger, [53] Fairbanks, ${ }^{[54]}$ and Rademann ${ }^{[55]}$ for the DMC-mediated $S$-glycosylations.

The DMC-mediated azidation was also studied carefully. Glycosyl azides are among the most important synthetic intermediates in sugar chemistry. The recent developments of 'click chemistry' [56] have dramatically increased the potential of sugars possessing an azide function as precursors for glycoarrays and glycoconjugates. With the addition of sodium azides to the reaction mixture of sugars and DMC, $\beta$-selective syntheses of various glycosyl azides 31 have been achieved (Scheme 14B). [57]

Dithiocarbamate is an important reagent used in radical reactions such as reversible addition-fragmentation chain transfer (RAFT) polymerization. ${ }^{58]}$ When the anomeric carbon is modified with a dithiocarbamate group, glycosyl radicals can be generated as a useful intermediate for a range of glycosylated reactions. ${ }^{[59]}$ In 2016, we proposed a protection-free approach towards the synthesis of glycosyl dithiocarbamates 32 in aqueous media by using dithiocarbamate salts as nucleophiles (Scheme 14C). ${ }^{[60]}$ In some cases, the carbon disulfide and secondary amines were used as the alternative nucleophiles for the designed dithiocarbamate structures.

The $S$-alkylthiosulfates (Bunte salts) have attracted our interest because they are stable, easy-to-handle crystalline substances, and they have proven to be highly versatile synthetic intermediates in organic reactions used by pharmaceutical and 


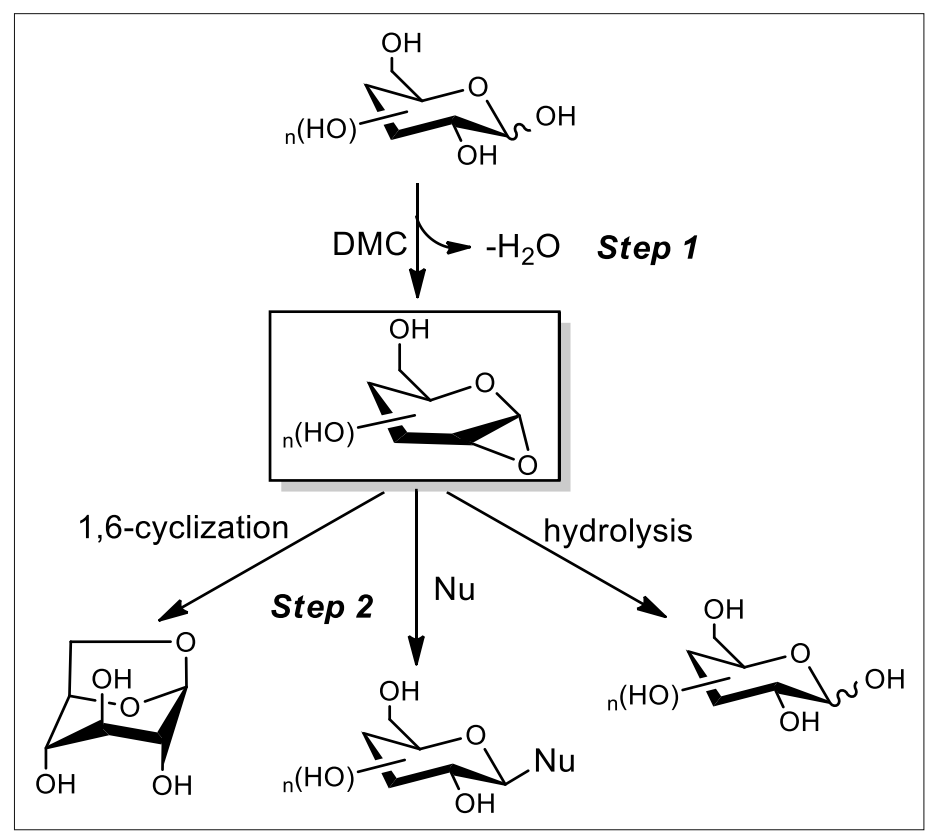

Scheme 13.

Dehydrative process in aqueous media: Formation of 1,2-anhydro sugars by intramolecular dehydrative cyclization (Step 1) and the subsequent nucleophilic attack at the anomeric position (Step 2).

surfactant manufacturers. ${ }^{[61]}$ In 2018, we expanded the DMC activation strategy to the synthesis of glycosyl-Bunte salts $\mathbf{3 3}$ with the addition of sodium thiosulfate $\left(\mathrm{Na}_{2} \mathrm{~S}_{2} \mathrm{O}_{3}\right)$ as nucleophile (Scheme 14D). [62] The application of glycosyl Bunte-salts has also been demonstrated with transformation reactions into other glycosyl compounds such as a 1-thio sugar $\mathbf{3 4}$, a glycosyl disulfide 35, a 1,6-anhydro sugar, and an $O$-glycoside.

Apart from our DMC strategy, Hocek and co-workers reported another approach for the formation of an 1,2-anhydro sugar 36 under modified Mitsunobu reaction conditions (Scheme 15).[63] They treated the 5-O-trityl protected ribose 37 with $\mathrm{P}(n-\mathrm{Bu})_{3}$ and 1,1'-(azodicarbonyl)dipiperidine (ADDP) in acetonitrile to produce 36 in nearly quantitative conversion. The oxirane ring opening using various nucleophiles revealed the versatile applicability of this anhydro intermediate. The 5-O-protected 1,2-anhydro sugar 36 was shown to be stable for at least one month in situ if kept under low humidity.

\subsection{First Detection of Unprotected 1,2-Anhydro Sugars}

The existence of unprotected 1,2-anhydro sugars has been rationalized by a theoretical study for levoglucosan formation from phenyl $\beta$-glucoside. ${ }^{[64]}$ Bennet and co-workers reported that the hydrolysis of $p$-nitrophenyl glycosides proceeds through a mechanism involving formation of 1,2-anhydro sugar intermediates. ${ }^{[65]}$ However, the direct experimental observation of unprotected 1,2-anhydro sugars had not been achieved in spite of their potential utilities in organic synthesis. In 2017, we were able to detect the unprotected 1,2-anhydro aldopyranose in the DMC-mediated reaction mixture for the first time with low-temperature NMR spectroscopy (Fig. 1). ${ }^{[4]}$ Proton NMR showed a doublet peak attributed to the anomeric proton at $5.10 \mathrm{ppm}$ with a small coupling constant of $2.0 \mathrm{~Hz}$. Compared with the ${ }^{13} \mathrm{C}$ NMR of $\alpha$-aldopyranose ${ }^{[66]}(\mathrm{C} 1$ at $92.9 \mathrm{ppm}, \mathrm{C} 2$ at $72.5 \mathrm{ppm}$ ), a $15 \mathrm{ppm}$ upfield shift was observed for the 1,2-anhydro aldopyranose (C1 at $78.7 \mathrm{ppm}, \mathrm{C} 2$ at 56.5 ppm), which clearly supports the existence of an oxirane ring, because the carbons in an oxirane ring are known to be detected at higher magnetic fields than those of other cyclic ethers due to the large ring distortion. The first observation and characterization of 1,2-anhydro sugars can be attributed to the mildness of the reaction conditions of the intramolecular dehydration reaction in aqueous media through the use of formamidinium-type agents.

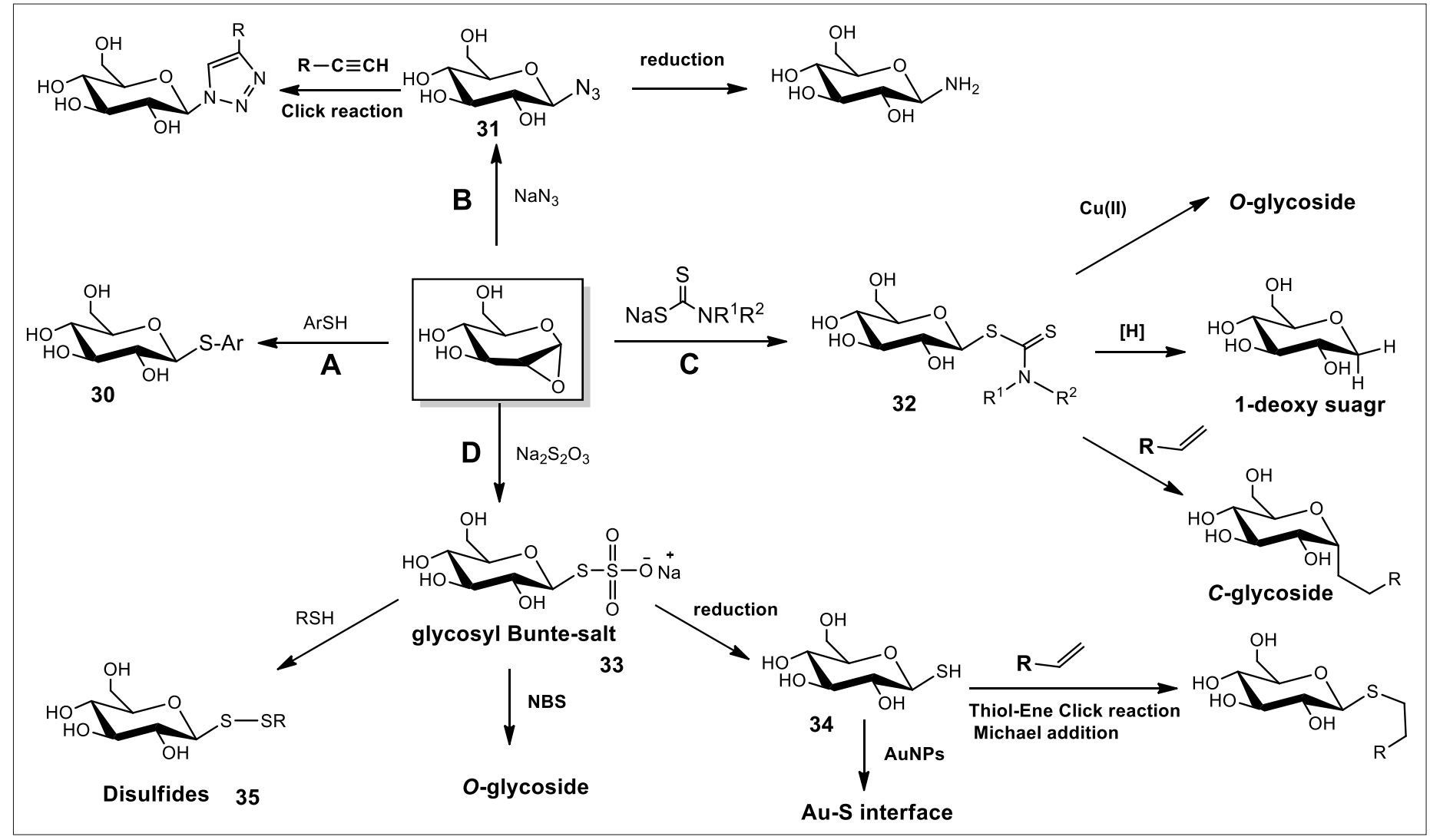

Scheme 14. Synthesis of various carbohydrate derivatives through unprotected 1,2-anhydro sugars. 
Scheme 15.

Formation of 1,2-anhydro sugars under modified Mitsunobu reaction conditions.

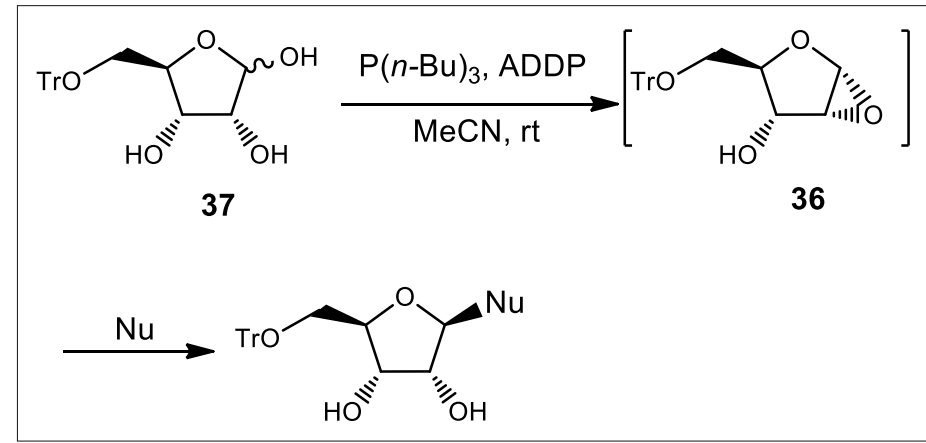

\section{Conclusion and Perspectives}

Protected and unprotected 1,2-anhydro sugars represent a class of important intermediates in the field of synthetic carbohydrate chemistry and can be used to achieve regio- and stereo-selective glycosylation reactions. A variety of advanced promoters have been developed to open the oxirane ring resulting in stereoselective glycosylation reactions. Encouraged by the direct synthesis of sugar oxazoline in aqueous media, the direct anomeric activation strategy allows for the preparation of unprotected 1,2-anhydro sugars in situ by using a chloroformamidinium-dehydrating agent and these intermediates can be detected using low-temperature NMR spectroscopy. A variety of attractive carbohydrate derivatives such as 1,6-anhydrosugars, glycosyl azides, thioglycosides and gly-

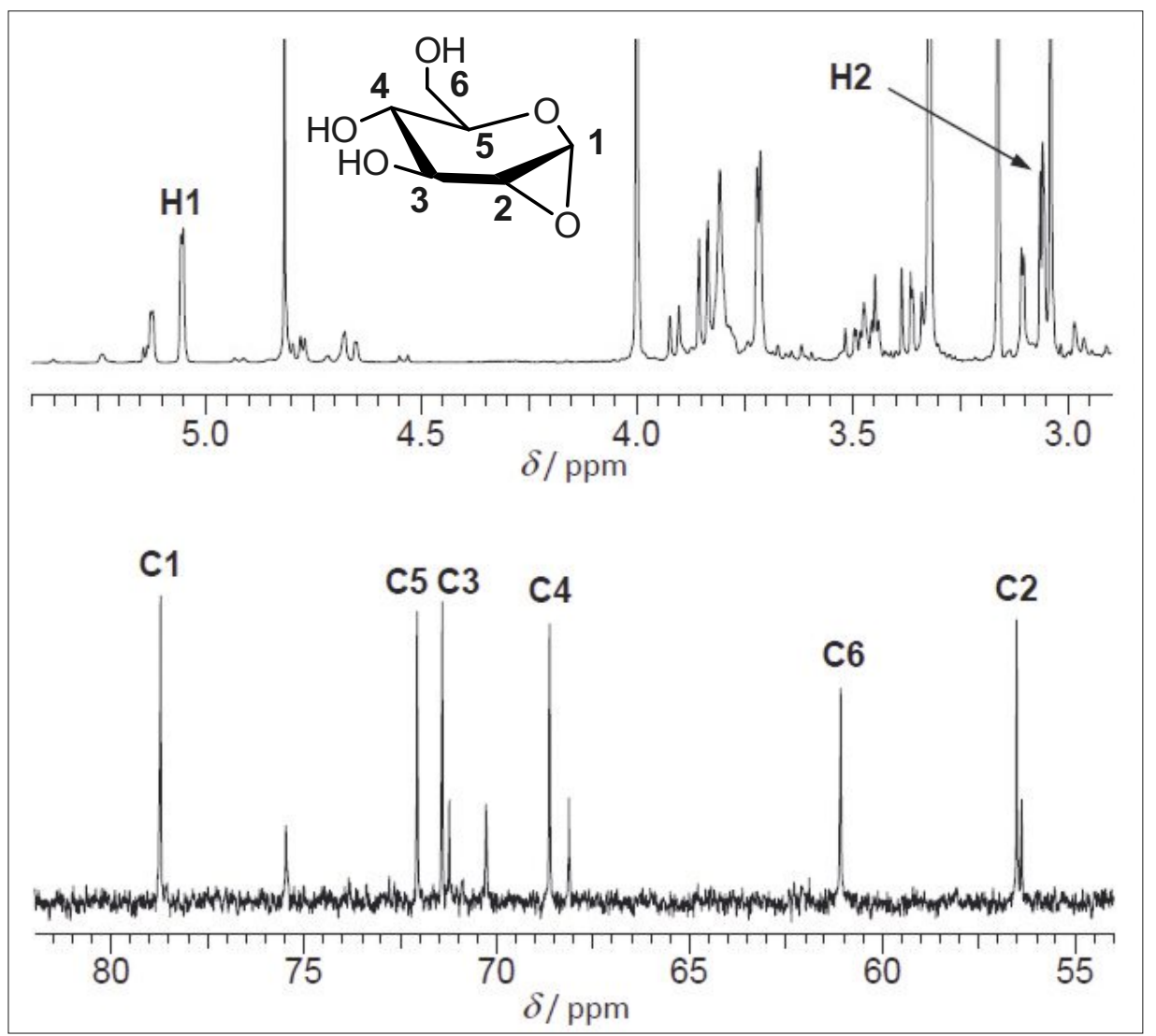

Fig. 1. ${ }^{1} \mathrm{H}$ and ${ }^{13} \mathrm{C}$ NMR spectra of the reaction mixture of glucose and $\mathrm{DMC}$ in $\mathrm{D}_{2} \mathrm{O} / \mathrm{CH}_{3} \mathrm{CN}$ at $-10{ }^{\circ} \mathrm{C}$.
Chun Huang and Dr. Naoki Yoshida for their contribution to the development of new glycosylation reactions described herein. This work was supported by a Grant-in-Aid for Scientific Research from the Ministry of Education, Sports, Science and Technology, Industrial Technology Research Grant Program.

Received: July 10, 2018

[1] P. Brigl, Hoppe-Seyler's Z. Physiol. Chem. 1922, 122, 245 .

[2] See for instance: R. L. Halcomb, S. J. Danishefsky, J. Am. Chem. Soc. 1989, 111, 6661.

[3] T. Tanaka, W. C. Huang, M. Noguchi, A Kobayashi, S. Shoda, Tetrahedron Lett. 2009, $50,2154$.

[4] K. Serizawa, M. Noguchi, G. Li, S. Shoda, Chem. Lett. 2017, 46, 1024.

[5] S. J. Sondheimer, H. Yamaguchi, C. Schuerch, Carbohydr. Res. 1979, 74, 327.

[6] a) F. Kong, J. Du, H. Shang, Carbohydr. Res. 1987, 162, 217; b) G. Yang, F. Kong, $J$. Carbohydr. Chem. 1994, 13, 909.

[7] a) E. Wu, Q. Wu, Carbohydr. Res. 1993, 250, 327; b) J. Ning, F. Kong, Carbohydr. Res. 2001 $330,165$.

[8] R. W. Murray, R. Jeyaraman, J. Org. Chem. 1985, 50, 2847.

[9] G. Bellucci, G. Catelani, C. Chiappe, F. D. Andrea, Tetrahedron Lett. 1994, 35, 8433.

[10] M. Cavicchioli, A. Mele, V. Montanari, G. Resnati, J. Chem. Soc., Chem. Commun. 1995, 901.

[11] E. Honda, D. Y. Gin, J. Am. Chem. Soc. 2002, $124,7343$.

[12] P. Cheshev, A. Marra, A. Dondoni, Carbohydr. Res. 2006, 341, 2714

[13] a) C. Liu, W. Yu, S. Li, C. Che, J. Org. Chem. 1998, 63, 7364; b) W. Chan, M. Wong, C. Che, J. Org. Chem. 2005, 70, 4226.

[14] a) X. Yu, J. Huang, W. Yu, C. Che, J. Am. Chem. Soc. 2000, 122, 5337; b) J. Zhang, C. Che, Org. Lett. 2002, 4, 1911; c) J. Zhang, J. Huang, C. Che, Chem. Eur. J. 2006, 12, 3020.

[15] a) R. U. Lemieux, G. Huber, J. Am. Chem. Soc. 1953, 75, 4118; b) R. U. Lemieux, G. Huber, $J$. Am. Chem. Soc. 1956, 78, 4117.

[16] M. P. Bardolph, G. H. Cloeman, J. Org. Chem. 1950, 15,169 .

[17] a) P. H. Seeberger, M. Eckhardt, C. E. Gutteridge, S. J. Danishefsky, J. Am. Chem. Soc. 1997, 119, 10064; b) P. H. Seeberger, X. Beebe, G. D. Sukenick, S. Pochapsky, S. J. Danishefsky, Angew. Chem. Int. Ed. Engl. 1997, 36, 491.

[18] Y. Du, F. Kong, J. Carbohydr. Chem. 1995, 14, 341.

[19] C. M. Timmers, S. C. N. Wigchert, M. A Leeuwenburgh, G. A. Marel, J. H. Boom, Eur. J. Org. Chem. 1998, 91.

[20] C. Ernst, W. Klaffke, Tetrahedron Lett. 2001, 42, 2973.

[21] Y. Li, P. Tang, Y. Chen, B. Yu, J. Org. Chem. 2008, 73, 4323.

[22] A. Nakagawa, M. Tanaka, S. Hanamura, D. Takahashi, K. Toshima, Angew. Chem. Int. Ed. 2015, 54, 10935

[23] C. Liu, D. Xiong, X. Ye, Tetrahedron Lett. 2016, 57, 1372.

[24] D. Somasundaram, K. K. Balasubramanian, S. Bhagavathy, Carbohydr. Res. 2017, 449, 95.

[25] Y. Yang, B. Yu, Chem. Rev. 2017, 117, 12281.

[26] S. Manabe, Y. Marui, Y. Ito, Chem. - Eur. J. 2003, 9, 1435

[27] S. P. Allwein, J. M. Cox, B. E. Howard, H. W. B. Johnson, J. D. Rainier, Tetrahedron 2002, 58 , 1997. 
[28] a) R. Chang, T. T. Vo, N. S. Finney, Carbohydr Res. 2006, 341, 1998; b) J. D. Rainier, J. M Cox, Org. Lett. 2000, 2, 2707; c) S. Xue, K.-Z. Han, L. He, Q.-X. Guo, Synlett 2003, 870; d) S. Wagschal, J. Guilbaud, P. Rabet, V. Farina, S. Lemaire, J. Org. Chem. 2015, 80, 9328; e) M. A. Leeuwenburgh, G. A. van der Marel, H. S. Overkleeft, J. H. J. van Boom, Carbohydr Chem. 2003, 22, 549; f) M. B. Tatina, A. K Kusunuru, D. Mukherjee, Org. Lett. 2015, 17 , 4624; g) P. Wipf, J. G. Pierce, N. Zhuang, Org. Lett. 2005, 7, 483.

[29] V. Bellosta, S. Czernecki, Carbohydr. Res. 1993, 244, 215.

[30] J. L. Chiara, E. Sesmilo, Angew. Chem. Int. Ed. 2002, 41, 3242 .

[31] J. D. Parrish, R. D. Little, Org. Lett. 2002, 4, 1439 .

[32] F. Zhu, J. Rodriguez, T. Yang, I. Kevlishvili, E. Miller, D. Yi, S. O'Neill, M. J. Rourke, P. Liu, M.A. Walczak, J. Am. Chem. Soc. 2017, 139, 17908.

[33] K. Chow, S. Danishefsky, J. Org. Chem. 1990, $55,4211$.

[34] J. Ning, F. Kong, Carbohydr. Res. 2000, 326, 235.

[35] Y. S. Reddy, A. P. J. Pal, P. Gupta, A. A. Ansari, Y. D. Vankar, J. Org. Chem. 2011, 76, 5972.

[36] R. T. Dere, A. Kumar, V. Kumar, X. Zhu, R. R. Schmidt, J. Org. Chem. 2011, 76, 7539.

[37] M. G. Nokwequ, C. M. Nkambule, D. M. Gammon, Carbohydr. Res. 2012, 359, 18

[38] a) P. Padungros, L. Alberch, A, Wei, Org. Lett. 2012, 14, 3380; b) P. Padungros, L. Alberch, A Wei, J. Org. Chem. 2014, 79, 2611.
[39] a) D. M. Gordon, S. J. Danishefsky, Carbohydr. Res. 1990, 111, 361; b) D. B. Berkowitz, S. J. Danishefsky, G. K. Schulte, J. Am. Chem. Soc. 1992, 114, 4518 .

[40] T. M. Flaherty, J. Gervay, Tetrahedron Lett. 1996, 37, 961.

[41] F. P. Boulineau, A. Wei, Org. Lett. 2002, 4, 2281.

[42] J. Castilla, I. Marín, M. I. Matheu, Y. Díaz, S. Castillón, J. Org. Chem. 2010, 75, 514.

[43] A. Pictet, P. Castan, Helv. Chim. Acta 1920, 3, 645.

[44] P. Brigl, R. Schinle, Ber. 1929, 62, 1716

[45] A. Dyfuerman, B. Lindberg, Acta Chem. Scand. 1950, 4, 878 .

[46] F. Micheel, A. Klemer, Chem. Ber. 1958, 91, 663.

[47] M. Noguchi, T. Tanaka, H. Gyakushi, A Kobayashi, S. Shoda, J. Org. Chem. 2009, 74, 2210.

[48] S. Shoda, Proc. Jpn. Acad., Ser. B 2017, 93, 125.

[49] M. Noguchi, T. Fujieda, W. C. Huang, M. Ishihara, A. Kobayashi, S. Shoda, Helv. Chim. Acta 2012, 95, 1928.

[50] T. Tanaka, T. Matsumoto, M. Noguchi, A Kobayshi, S. Shoda, Chem. Lett. 2009, 38, 458.

[51] N. Yoshida, M. Noguchi, T. Tanaka, T. Matsumoto, N. Aida, M. Ishihara, A. Kobayashi, S. Shoda, Chem. Asian J. 2011, 6, 1876.

[52] N. Yoshida, T. Fujieda, A. Kobayashi, M. Ishihara, M. Noguchi, S. Shoda, Chem. Lett. 2013, 42, 1038.

[53] A. Novoa, S. Barluenga, C. Serba, N. Winssinger, Chem. Commun. 2013, 49, 7608.
[54] S. R. Alexander, A. J. Fairbanks, Org. Biomol. Chem. 2016, 14, 6679 .

[55] S. Koehling, M. P. Exner, S. Nojoumi, J. Schiller, N. Budisa, J. Rademann, Angew. Chem. Int. Ed. 2016, 55, 15510.

[56] H. C. Kolb, M. G. Finn, K. B. Sharpless, Angew. Chem. Int. Ed. 2001, 40, 2004.

[57] T. Tanaka, H. Nagai, M. Noguchi, A. Kobayashi, S. Shoda, Chem. Commun. 2009, 45, 3378 .

[58] J. Chiefari, R. T. A. Mayadunne, C. L. Moad, G. Moad, E. Rizzardo, A. Postma, M. A. Skidmore, S. H. Thang, Macromolecules 2003, 36, 2273.

[59] a) Q. Wan, A. Lubineau, M. C. Scherrmann, J. Carbohyd. Chem. 2004, 23, 83; b) C. McMaster, R. N. Bream, R. S. Grainger, Org. Biomol. Chem. 2012, 10, 4752.

[60] G. Li, M. Noguchi, H. Kashiwagura, Y. Tanaka, K. Serizawa, S. Shoda, Tetrahedron Lett. 2016, 57, 3529.

[61] H. Distler, Angew. Chem. Int. Ed. Engl. 1967, 6, 544.

[62] Y. Meguro, M. Noguchi, G. Li, S. Shoda, Org. Lett. 2018, 20, 76

[63] a) A. M. Downey, C. Richter, R. Pohi, R. Mahrwald, M. Hocek, Org. Lett. 2015, 17, 4604; b) A. M. Downey, R. Pohi, J. Toithová, M. Hocek, Chem. Eur. J. 2017, 23, 3910.

[64] S. Aono, T. Hosoya, S. Sakaki, Phys. Chem. Chem. Phys. 2013, 15, 6368.

[65] G. Speciale, M. Farren-Dai, F. S. Shidmoossavee, S. J. Williams, A. J. Bennet, $J$. Am. Chem. Soc. 2016, 138, 14012.

[66] P. Collins, R. Ferrier, 'Monosaccharides', Wiley, Chichester, 1995, p. 535. 